v.3 n.14 (2022)

Recebido: 30/11/2021

Aceito: 07/12/2021

\title{
Suspensões cirúrgicas em um hospital público do Distrito Federal
}

\author{
Lorena Magalhães Guedes Ferreira Brito ${ }^{1}$ \\ Ruth Silva Matos ${ }^{2}$ \\ Raquel Elisa de Almeida ${ }^{3}$ \\ Mirce Meire Gonçalves de Sousa Wilk ${ }^{4}$ \\ Jacqueline Ramos de Andrade Antunes Gomes ${ }^{5}$ \\ Lauane Rocha Itacarambi ${ }^{6}$ \\ Gleyce Mikaelle Costa Quirino ${ }^{7}$ \\ Luciane Fermon Damasceno Ribeiro ${ }^{8}$ \\ Aline Maria Campos de Melo 9 \\ Liliane Travassos Cavalcanti de Souza ${ }^{10}$ \\ Hysnara Gomes Lima de Lucena ${ }^{11}$ \\ Raquel Barcellos Marques Schiffer ${ }^{12}$
}

\footnotetext{
${ }^{1}$ Enfermeira, pós-graduanda do Programa de Residência Uniprofissional de Enfermagem em Centro Cirúrgico ESCS/FEPECS/SES-DF.

${ }^{2}$ Enfermeira, pós-graduanda do Programa de Residência Uniprofissional de Enfermagem em Centro Cirúrgico ESCS/FEPECS/SES-DF.

${ }^{3}$ Enfermeira da Secretaria de Saúde do Distrito Federal, Tutora do Programa de Residência Uniprofissional de Enfermagem em Centro Cirúrgico - ESCS/FEPECS/SES-DF. Mestre em Enfermagem pela Universidade de Brasília (UnB).

${ }^{4}$ Enfermeira da Secretaria de Saúde do Distrito Federal, Tutora do Programa de Residência Uniprofissional de Enfermagem em Centro Cirúrgico - ESCS/FEPECS/SES-DF. Mestrando Ciências da Saúde pela Fundação de Ensino e Pesquisa em Ciências da Saúde (FEPECS).

${ }^{5}$ Enfermeira Coordenadora do Programa de Residência de Enfermagem em Centro Cirúrgico ESCS/FEPECS/SES/DF

${ }^{6}$ Enfermeira, pós-graduanda do Programa de Residência Uniprofissional de Enfermagem em Centro Cirúrgico ESCS/FEPECS/SES-DF

${ }^{7}$ Enfermeira, pós-graduanda do Programa de Residência Uniprofissional de Enfermagem em Centro Cirúrgico ESCS/FEPECS/SES-DF

${ }^{8}$ Enfermeira Preceptora do Programa de Residência de Enfermagem em Centro Cirúrgico ESCS/FEPECS/SES/DF

${ }^{9}$ Enfermeira Tutora do Programa de Residência de Enfermagem em Centro Cirúrgico ESCS/FEPECS/SES/DF

${ }^{10}$ Enfermeiro Tutor do Programa de Residência de Enfermagem em Centro Cirúrgico ESCS/FEPECS/SES/DF

${ }^{11}$ Enfermeiro Preceptora do Programa de Residência de Enfermagem em Centro Cirúrgico ESCS/FEPECS/SES/DF

${ }^{12}$ Enfermeiro Preceptora do Programa de Residência de Enfermagem em Centro Cirúrgico ESCS/FEPECS/SES/DF
} 


\title{
RESUMO
}

Objetivo: Investigar a incidência e as causas de suspensões cirúrgicas em uma unidade de Centro Cirúrgico de um hospital público de médio porte localizado no Distrito Federal. Metodologia: Trata-se de uma pesquisa retrospectiva, descritiva e quantitativa realizada a partir dos prontuários eletrônicos dos pacientes e registros hospitalares. Resultados: Das 1.197 cirurgias eletivas programadas no período de janeiro a julho de 2019, 235 foram canceladas, totalizando uma taxa de suspensão cirúrgica de 19,6\%. O principal motivo de suspensão foi relacionado ao paciente $(37,4 \%)$, como as condições clínicas desfavoráveis e o não comparecimento. Conclusão: A incidência de suspensão cirúrgica no hospital cenário do estudo é semelhante à taxa divulgada em outros estudos em hospitais públicos. Para evitar desnecessários cancelamentos faz-se necessário o monitoramento contínuo da incidência e dos motivos de suspensões cirúrgicas como um importante indicador de qualidade.

Palavras-chaves: Procedimentos Cirúrgicos Operatórios; Enfermagem Perioperatória; Indicadores de Qualidade em Assistência à Saúde; Centros Cirúrgicos.

\section{Surgical suspensions in a public hospital in the Federal District}

\begin{abstract}
Objective: Investigate the incidence and causes of surgical suspensions in a Surgical Center unit of a medium-sized public hospital located in the Federal District. Methodology: This is a retrospective, descriptive and quantitative research carried out from the electronic medical records of patients and hospital records. Results: Of the 1,197 elective surgeries scheduled from January to July 2019, 235 were canceled, totaling a surgical suspension rate of $19.6 \%$. The main reason for suspension was related to the patient (37.4\%), such as unfavorable clinical conditions and non-attendance. Conclusion: The incidence of surgical suspension in the study hospital is similar to the rate reported in other studies in public hospitals. To avoid unnecessary cancellations, it is necessary to continuously monitor the incidence and reasons for surgical suspensions as an important quality indicator.
\end{abstract}

Keywords: Surgical Procedures, Operative; Perioperative Nursing; Quality Indicators, Health Care; Surgicenters.

\section{INTRODUÇÃO}

O Centro Cirúrgico (CC) constitui uma das unidades mais complexas do ambiente hospitalar, repleta de normas e rotinas a serem seguidas para o bom funcionamento do serviço. O bom desempenho da unidade está diretamente relacionado com a qualidade de seus próprios processos e com os processos dos serviços que o apoiam ${ }^{1}$.

O processo de trabalho do enfermeiro em CC é direcionado para o gerenciamento da unidade, com o propósito de garantir as melhores condições para o desenvolvimento da prática cirúrgica, em prol do paciente. Embora o trabalho em saúde seja coletivo, é o profissional enfermeiro que responde institucionalmente pela qualidade do serviço ${ }^{2}$. 
Entende-se como qualidade um conjunto de atributos que inclui: nível de excelência profissional, uso racional de recursos, maior benefício e menor risco para o paciente e alto grau de satisfação por parte dos usuários ${ }^{3}$.

Ao longo dos anos vem crescendo a preocupação com a gestão da qualidade dos serviços prestados pela instituição de saúde ao paciente. Deste modo, é fundamental que sejam empregados instrumentos específicos que norteiem os profissionais de saúde, em especial o enfermeiro, na busca de níveis mais elevados de qualidade, proporcionando serviços que atendam às necessidades dos cidadãos com segurança ${ }^{4}$.

A avaliação da qualidade dos serviços no $\mathrm{CC}$ se dá por meio de indicadores de qualidade focados na estrutura, nos processos e nos resultados dos serviços oferecidos ${ }^{5}$. Os indicadores de qualidade devem possuir características importantes, tais como validade causal, pertinência, viabilidade de cálculo, simplicidade e, também especificidade, para então serem devidamente monitorados, analisados ao longo do tempo e comparados à realidade interna e externa das organizações de saúde ${ }^{6}$. Para efeito desta pesquisa, cabe destacar a taxa de suspensão cirúrgica que é um importante indicador de processo no $\mathrm{CC}^{4}$.

A suspensão cirúrgica é definida como o rompimento de qualquer operação que estava programada e que não foi realizada na data agendada, sendo seus motivos normalmente identificados no dia útil posterior ${ }^{5,6}$.

Estudos com o objetivo de investigar a incidência de suspensão cirúrgica e de identificar seus motivos têm sido realizados em diversos lugares do mundo. Questões organizacionais das instituições de saúde são indicadas como o principal motivo para elevada taxa de suspensão cirúrgica nos países como Austrália, Irlanda, México, Estados Unidos e Reino Unido ${ }^{7}$. No Brasil, os dados são alarmantes, variam de $6,79 \%^{8}$ a $33,8 \%{ }^{9}$.

Condição clínica desfavorável; não comparecimento para internação; falta de exames previamente solicitados; falhas no pré-operatório; ausência e atrasos da equipe cirúrgica; 
ultrapassagem do tempo cirúrgico; mudança de conduta médica; falta de insumos necessários; falta de sala cirúrgica; falta de leitos disponíveis para internação; erros de agendamento e falhas de comunicação entre equipe médica, CC e unidades de internação, são fatores de suspensão de cirurgia evidenciados na literatura por diversos autores ${ }^{5-10}$.

Além dos aspectos clínicos e operacionais, cabe mencionar algumas condições inerentes ao indivíduo, uma vez que a programação de uma cirurgia é algo que acarreta uma série de inconveniências, tais como mudança de rotina e preparo emocional para enfrentar uma situação sobre a qual, muitas vezes, não possui informações suficientemente precisas ${ }^{10}$.

Para muitos pacientes e familiares a cirurgia é um fator de ansiedade e estresse devido ao temor da anestesia somado aos possíveis riscos em qualquer procedimento desta natureza $^{11}$. A suspensão deste acontecimento intensifica esse fator e merece atenção especial do enfermeiro bem como de toda equipe cirúrgica ${ }^{12}$.

Sobre a perspectiva organizacional, a suspensão de cirurgia gera custos e gastos hospitalares, além de interferir na operacionalização do trabalho da equipe. Vale ressaltar que a suspensão cirúrgica é uma falha que sucede ao não atendimento aos requisitos do planejamento administrativo da unidade ${ }^{12,}{ }^{13}$, sendo um problema a ser manejado pelo enfermeiro, quando recorrente ${ }^{6}$.

Portanto, para subsidiar as tomadas de decisão dos gestores é essencial conhecer a ocorrência e as principais causas de suspensões cirúrgicas a fim de melhorar a qualidade do serviço, estimar a utilização de recursos e amenizar o sofrimento do paciente e família.

Diante do exposto, a presente pesquisa visa responder a seguinte questão: Qual a incidência de suspensão de procedimentos cirúrgicos e quais os motivos para a ocorrência desses cancelamentos cirúrgicos?

Mediante isso, pretende-se investigar a incidência e as causas de suspensões cirúrgicas em um CC de um hospital público de médio porte localizado no Distrito Federal, assim como 
relacionar as suspensões com as características demográficas do paciente (sexo e idade) e as especialidades cirúrgicas.

\section{METODOLOGIA}

Trata-se de um estudo retrospectivo, de natureza descritiva e, em razão de sua maior precisão e confiabilidade, foi utilizada a abordagem quantitativa, pois seus resultados são passíveis de generalização, principalmente quando as amostras pesquisadas representam, com fidelidade, a população de onde foram retiradas e por isso são mais indicadas para o planejamento de ações coletivas ${ }^{14}$.

O estudo foi realizado no Centro Cirúrgico (CC) de um hospital público de médio porte localizado no Distrito Federal, que presta assistência médico-hospitalar de média complexidade, sendo referência no Sistema Único de Saúde (SUS).

O CC do hospital cenário do estudo realiza uma média de 180 cirurgias eletivas ao mês, e dispõe de cinco salas de cirurgia e seis leitos para Sala de Recuperação Pós-Anestésica (SRPA). A programação cirúrgica eletiva é desenvolvida no período das 7 às $19 \mathrm{~h}$, de segunda a sexta-feira. Além disso, realiza uma média de 100 cirurgias de urgência mensalmente.

No CC em questão são atendidos pacientes cirúrgicos das especialidades Cirurgia Pediátrica, Ginecologia, Reprodução Humana, Mastologia, Otorrinolaringologia, Urologia, Dermatologia, Proctologia, Oftalmologia e Odontologia.

Os dados foram coletados a partir de fontes secundárias, sendo elas prontuários eletrônicos dos pacientes e registros hospitalares. Para a coleta foi utilizado um roteiro estruturado formulado pela própria pesquisadora, contendo as seguintes variáveis: sexo do paciente, idade, data do procedimento cirúrgico, especialidade médica e motivo da suspensão.

A população estudada foi composta por cirurgias eletivas agendadas entre janeiro e julho de 2019 no hospital cenário do estudo, o que correspondeu a 1.197 procedimentos cirúrgicos. Os critérios de exclusão foram os procedimentos cirúrgicos de urgência e/ou 
emergência, cirurgias de pacientes que não tinham em seus prontuários os dados a serem analisados ou que os registros hospitalares de suspensão cirúrgica não haviam sido preenchidos.

Os dados foram analisados utilizando o programa IBM Statistical Package for Social Sciences (SPSS) versão 21.0, através de estatística descritiva, ou seja, frequência absoluta e relativa.

Este projeto foi devidamente submetido ao Comitê de Ética em Pesquisa da Fundação de Ensino e Pesquisa em Ciências da Saúde - CEP/FEPECS, e aprovado sob o número CAAE 15903119.4.0000.5553, conforme preconiza as normas da Resolução CNS nº 466, de 12 de dezembro de 2012.

\section{RESULTADOS}

No período estudado, entre janeiro e julho de 2019, foram programadas 1.197 cirurgias, das quais foram realizadas 962 e suspensas 235, totalizando uma taxa de suspensão cirúrgica de 19,6\%. A taxa de suspensão cirúrgica é o resultado da divisão da quantidade de cirurgias suspensas pelo total de cirurgias programadas em determinado período e multiplicado por 100, conforme as normas do Ministério da Saúde do Brasil ${ }^{7}$.

A distribuição de suspensões foi de 125 cirurgias canceladas para mulheres $(53,19 \%)$ e 110 para homens $(46,81 \%)$. Em relação à idade, foi encontrado um maior número de suspensão nos indivíduos com idade superior ou igual a 19 anos e 59 anos de idade $(48,5 \%)$ (Tabela 1).

Tabela 1 - Distribuição das cirurgias suspensas por sexo, faixa etária e especialidade (n=235)

\begin{tabular}{lcc}
\hline Variáveis & N & \% \\
\hline Sexo & & \\
Feminino & 125 & 53,2 \\
Masculino & 110 & 46,8
\end{tabular}

Faixa etária 
Criança ( $\leq 12$ anos $)$

Adolescente (13 a 18 anos)

Adulto (19 a 59 anos)

Idoso ( $\geq 60$ anos)

Especialidade

Cirurgia Pediátrica

Dermatologia

Ginecologia

Mastologia

Odontologia

Oftalmologia

Otorrinolaringologia

Proctologia

Urologia

Fonte: Elaborada pela própria autora.

A especialidade cirúrgica em que ocorreu quantidade maior de suspensão foi a ginecologia $(34,9 \%)$, seguidas da cirurgia pediátrica $(28,9 \%)$ e urologia $(14 \%)$. No entanto, ao se analisar a suspensão conforme os agendamentos por especialidade (relação entre o número de cirurgias canceladas por especialização e o número de cirurgias programadas nessa área), aquela em que apresentou maior taxa de suspensão cirúrgica foi a otorrinolaringologia $(36,5 \%)$, seguidas da odontologia (30\%) e urologia (22,9\%) (Tabela 2).

Tabela 2 - Taxa de suspensão conforme sexo, idade e especialidade

\begin{tabular}{|c|c|c|c|}
\hline Variáveis & $\begin{array}{c}\text { Agendadas } \\
(n=1197)\end{array}$ & $\begin{array}{c}\text { Suspensas } \\
(n=235)\end{array}$ & $\begin{array}{c}\text { Taxa de } \\
\text { Suspensão }(\%)\end{array}$ \\
\hline \multicolumn{4}{|l|}{ Sexo } \\
\hline Feminino & 729 & 125 & 17,1 \\
\hline Masculino & 468 & 110 & 23,5 \\
\hline \multicolumn{4}{|l|}{ Faixa etária } \\
\hline Criança $(\leq 12$ anos $)$ & 358 & 86 & 24,0 \\
\hline Adolescente (13 a 18 anos) & 66 & 16 & 24,2 \\
\hline Adulto (19 a 59 anos) & 664 & 114 & 17,2 \\
\hline Idoso $(\geq 60$ anos $)$ & 109 & 19 & 17,4 \\
\hline \multicolumn{4}{|l|}{ Especialidade } \\
\hline Cirurgia Pediátrica & 313 & 68 & 21,7 \\
\hline Dermatologia & 64 & 5 & 7,8 \\
\hline Ginecologia & 495 & 82 & 16,6 \\
\hline
\end{tabular}




$\begin{array}{lccc}\text { Mastologia } & 45 & 10 & 22,2 \\ \text { Odontologia } & 10 & 3 & 30,0 \\ \text { Oftalmologia } & 71 & 15 & 21,1 \\ \text { Otorrinolaringologia } & 52 & 19 & 36,5 \\ \text { Proctologia } & 3 & 0 & 0,0 \\ \text { Urologia } & 144 & 33 & 22,9\end{array}$

Fonte: Elaborada pela própria autora.

Das cirurgias programadas, setenta e setes foram suspensos sem apresentar justificativas e uma reagendada sem descrição do motivo; sendo assim, foram agrupadas dentro do motivo de cirurgias suspensas "não informado", totalizando $78(33,2 \%)$ cirurgias canceladas sem especificação do seu real motivo.

Os demais motivos das cirurgias suspensas foram organizados em quatro categorias: a) relacionados ao paciente, que corresponde ao seu não comparecimento, condição clínica não favorável ao procedimento cirúrgico, falta de jejum, falta de documentação, recusa à realização de cirurgia ou óbito do paciente; b) relacionados à unidade, que se refere à gestão do bloco cirúrgico, tais como erro na programação cirúrgica, substituição da cirurgia por outra de emergência, falta de sala cirúrgica, falta de leitos disponíveis para internação ou falta de exames; c) relacionados aos recursos humanos, correspondendo à mudança de conduta médica, avanço de horário ou carência de algum profissional da equipe cirúrgica; d) referentes aos recursos materiais, devido à ausência de algum material cirúrgico ou da farmácia que impeça a realização do procedimento cirúrgico.

O motivo de suspensão cirúrgica mais observado no estudo foi relacionado ao paciente (37,4\%), sendo $43(18,3 \%)$ cirurgias canceladas devido a condição clínica desfavorável e 38 $(16,2 \%)$ por não comparecimento. As causas relacionadas aos recursos humanos representaram $15,7 \%$, com $13(5,5 \%)$ suspensões por falta de anestesiologista e $11(4,7 \%)$ por avanço de horário. Em relação aos motivos pertencentes à unidade foram observadas 27 $(11,5 \%)$ cirurgias canceladas por erro na programação cirúrgica; no tocante aos recursos materiais, duas $(0,9 \%)$ cirurgias suspensas (Tabela 3$)$. 
Tabela 3 - Distribuição das causas de suspensões cirúrgicas relacionadas ao paciente, à unidade, aos recursos humanos e aos recursos materiais

\begin{tabular}{lcc}
\hline Causas & $\mathbf{N}$ & $\mathbf{\%}$ \\
\hline Relacionadas ao paciente & $\mathbf{8 8}$ & $\mathbf{3 7 , 4}$ \\
Condição clínica desfavorável & 43 & 18,3 \\
Não comparecimento & 38 & 16,2 \\
Falta de jejum & 3 & 1,3 \\
Recusa à realização de cirurgia & 2 & 0,9 \\
Falta de documentação & 1 & 0,4 \\
Outros & 1 & 0,4 \\
& & \\
Relacionadas à unidade & $\mathbf{3 0}$ & $\mathbf{1 2 , 8}$ \\
Erro na programação cirúrgica & 27 & 11,5 \\
Falta de exames & 2 & 0,9 \\
Prioridade para emergência & 1 & 0,4 \\
& & \\
Relacionadas aos recursos humanos & $\mathbf{3 7}$ & $\mathbf{1 5 , 7}$ \\
Falta de anestesiologista & 13 & 5,5 \\
Avanço de horário & 11 & 4,7 \\
Falta de cirurgião & 9 & 3,8 \\
Mudança de conduta médica & 4 & 1,7 \\
Relacionadas aos recursos materiais & & $\mathbf{0 , 9}$ \\
Falta de material na farmácia & $\mathbf{2}$ & 0,4 \\
Não especificado & 1 & 0,4 \\
Não informado & 1 & $\mathbf{3 3 , 2}$ \\
\hline TOTAL & $\mathbf{7 8}$ & $\mathbf{1 0 0 , 0}$ \\
\hline Font Elaborapela prona & $\mathbf{2 3 5}$ &
\end{tabular}

Fonte: Elaborada pela própria autora.

\section{DISCUSSÃO}

O hospital cenário do estudo apresentou taxa de suspensão de 19,6\%. Este dado é semelhante à taxa divulgada em outra pesquisa, onde foi identificada uma taxa global de $19,5 \%$ em um hospital de ensino de médio porte localizado no nordeste brasileiro 5 . Outro estudo semelhante revelou alto índice de suspensão cirúrgica, correspondendo a uma taxa de $33,8 \%{ }^{9}$. Também foram encontradas taxas entre $6,79 \%$ e $30,6 \%$ em mais outras investigações $^{6-8,10,11,15}$. 
Não se tem conhecimento de um padrão aceitável para o indicador de taxa de suspensão cirúrgica e o seu controle deve ir além do monitoramento matemático do indicador; assim sendo, as ações gerenciais devem ser estratégicas e sistemáticas para a redução das $\operatorname{taxas}^{6}$, visando a qualidade dos serviços da unidade de atendimento do Centro Cirúrgico bem como da instituição.

Evidenciou-se predominância de cirurgias marcadas para serem realizadas em pacientes do sexo feminino, devido aos tipos de procedimentos que são realizados no hospital analisado, acompanhado frequentemente por intervenções cirúrgicas ginecológicas; por consequência, houve maior número de cancelamento de cirurgias em mulheres. No entanto, a taxa de suspensão cirúrgica foi maior no sexo masculino $(23,5 \%)$ (Tabela 2). Esse resultado difere de outras pesquisas, onde não teve alteração significativa entre os sexos, em ambas análises $^{10,11,15}$.

Em relação à idade, verificou-se maior incidência de cirurgias agendadas para adultos (entre 19 e 59 anos). Quando se analisa o número absoluto de suspensões, pode-se perceber que essa mesma faixa etária também são as que mais sofreram o cancelamento do procedimento, corroborando com outras pesquisas realizadas em hospitais públicos do estado de São Paulo ${ }^{8,11}$. Cabe destacar, que a taxa de suspensão de acordo com os agendamentos em crianças e adolescentes equivaleram a $24,0 \%$ e $24,2 \%$, respectivamente, e em idosos, $17,4 \%$, dados superiores à suspensão por agendamento em adultos (17,2\%) (Tabela 2).

No que tange à suspensão por especialidade cirúrgica, a ginecologia apresentou o maior valor absoluto $(34,9 \%)$, já em outros estudos não houve relevância no cancelamento de cirurgias desta especialidade, constatando-se taxas de 3,8\%, 7,09\% e 9,4\% $\%^{5-7}$. Em duas outras investigações, o maior número de suspensão pertenceu à cirurgia pediátrica ${ }^{5,8}$, entretanto neste estudo tal especialidade apresentou a segunda maior frequência. 
Por outro lado, na Tabela 2, pode-se observar que na distribuição dos agendamentos por especialidade, a maior taxa de suspensão foi encontrada nas cirurgias de otorrinolaringologia (36,5\%). Essa informação também foi encontrada em outra pesquisa, onde ao se analisar a taxa de suspensões cirúrgicas em cada especialidade, a otorrinolaringologia foi o campo que mais suspendeu os procedimentos, cancelando $21 \%$ de suas cirurgias eletivas ${ }^{15}$. A urologia, que neste estudo teve o terceiro maior índice $(22,9 \%)$, também foi o terceiro maior em outro trabalho, ao se aplicar a mesma análise estatística, com $36,6 \%$ de suas cirurgias suspensas ${ }^{10}$. Nenhuma cirurgia da proctologia foi suspensa, ao contrário do estudo supracitado, onde esta especialidade representou a maior taxa, com $43,1 \%{ }^{10}$

Analisando-se as causas de suspensões cirúrgicas, constatou-se que a maioria das ocorrências estavam relacionadas ao paciente, em que 18,3\% cirurgias foram canceladas por condição clínica desfavorável, tendo como, neste estudo, a principal causa para a não realização do procedimento cirúrgico. Identificou-se principalmente maior ocorrência nas especialidades da ginecologia e da cirurgia pediátrica.

Uma pesquisa envolvendo um hospital público no estado de São Paulo constatou uma maioria de índices justificados por condições clínicas desfavoráveis (48,33\%) nas quais incluem todos os eventos que envolvem Infecção das Vias Aéreas Superiores (IVAS), pneumonias, tosse produtiva e queda de saturação de $\mathrm{O}_{2}$, Infecção de Trato Urinário (ITU) e outros, não caracterizados, mas que inviabiliza a realização da cirurgia ${ }^{8}$. Entretanto, em outros trabalhos científicos realizados em hospitais públicos dos estados do Paraná e Pernambuco foram encontrados resultados inferiores a cinco por cento, $4,29 \%$ e $4,9 \%$, respectivamente ${ }^{6,7}$.

Outra razão de cancelamento cirúrgico relacionado ao paciente, identificado no hospital cenário de estudo, foi o não comparecimento, representando 16,2\% da amostra, sobretudo na urologia. Em Pernambuco foram observadas 23,2\% cirurgias canceladas pelo 
absenteísmo do paciente, sendo a principal causa de cancelamento constatado pelos autores ${ }^{7}$. Ainda segundo esse estudo, o número elevado de suspensão por não comparecimento é justificado por não existir um serviço de busca ativa para confirmar a presença do paciente à internação, poucos dias antes da data agendada ${ }^{7}$.

Em seguida aparecem motivos relacionados a erro na programação cirúrgica (11,5\%). Estudo realizado em hospital de ensino público do interior do Paraná revelou que 21,45\% das suspensões ocorreram devido à ineficiência do planejamento cirúrgico, as quais podem ser evitadas com o uso de meios e instrumentos de gestão atrelados às competências advindas da organização do trabalho pelos profissionais ${ }^{6}$.

Nesse mesmo estudo realizado no Paraná, a "falta de horário" foi um motivo associado ao planejamento cirúrgico ${ }^{6}$. Entende-se que isto ocorre em razão do excesso de agendamentos de cirurgias para o mesmo dia sem sequer analisar a complexidade do procedimento. Contudo, reuniões semanais são realizadas no hospital cenário de estudo a fim de planejar e, se necessário, remanejar as operações cirúrgicas com vista à redução da taxa de suspensão cirúrgica. Portanto, o "avanço de horário", presente como causa de cancelamento de cirurgia nesse estudo, representa um motivo relacionado aos recursos humanos, devido ao tempo excedido da cirurgia anterior ou atraso da equipe em iniciar o procedimento cirúrgico.

A redução dos índices de cancelamento está atrelada a capacidade de liderança e de planejamento eficaz do enfermeiro responsável por um centro cirúrgico ${ }^{11}$. A literatura recomenda a realização de consulta pré-anestésica ou pré-operatória, a confirmação telefônica do comparecimento dos pacientes dois dias antes do procedimento e a reestruturação do processo de trabalho envolvendo equipe de saúde, tecnologia de informação e profissionais de nível médio como estratégias para minimizar o cancelamento de cirurgias ${ }^{8}$.

Dessa forma, investigar a incidência e as causas de suspensões cirúrgicas, como um importante indicador de qualidade, permite que os profissionais avaliem e analisem os 
aspectos necessários ao atendimento para melhor planejamento administrativo e cirúrgico. Ressalta-se a importância do preenchimento do motivo da suspensão no mapa cirúrgico para averiguação de todas as causas, visto que 33,2\% cirurgias foram canceladas sem especificação do motivo, porém isso pode ser um desafio aos serviços públicos devido aos modelos de gestão arcaicos ainda presentes nessas instituições ${ }^{6}$. Todavia, a conscientização do profissional de enfermagem em registrar corretamente os dados e atualizá-los periodicamente permite a elaboração de estratégias voltadas para a realidade do setor.

\section{CONCLUSÃO}

A incidência de suspensão cirúrgica no hospital cenário do estudo é semelhante à taxa divulgada em outros estudos em hospitais públicos, tendo como principal causa problemas relacionados ao paciente, como as condições clínicas desfavoráveis e o não comparecimento do paciente. Também estiveram presentes problemas relacionados aos profissionais membros da equipe cirúrgica e fatores relacionados à instituição.

Além das suspensões cirúrgicas ocasionarem desgastes emocionais para os pacientes, familiares e profissionais de saúde, geram custos maiores para a instituição assim como aumento de riscos de infecção hospitalar, pelo fato de o paciente ter uma maior permanência de internação. Isso pode comprometer o bom andamento do processo de trabalho e diminuir a qualidade da assistência em saúde prestada ao usuário.

Para evitar desnecessários cancelamentos, esforços devem ser feitos para melhorar a relação custo-benefício por meio de planejamento cuidadoso e utilização eficiente dos recursos hospitalares disponíveis.

Nessa perspectiva, o conhecimento dos motivos de suspensões cirúrgicas auxilia no embasamento para tomada de decisão dos gestores e trabalhadores da instituição visando reduzir a incidência de cancelamento de procedimentos cirúrgicos e, consequentemente, reduzir os transtornos causados aos pacientes e familiares e os custos hospitalares. 


\section{REFERÊNCIAS}

1. Duarte IG, Ferreira DP. Uso de Indicadores na Gestão de um Centro Cirúrgico. Rev. adm. saúde; [Internet] Vol. 8, No 31 - Abr-Jun, 2006. [Acesso em 19 jul 2021] Disponível em: http://bases.bireme.br/cgi-

bin/wxislind.exe/iah/online/?IsisScript=iah/iah.xis\&src=google \&base=LILACS\&lang=p\&nex tAction=lnk\&exprSearch=446427\&indexSearch=ID.

2. Morgan W, Bernardino E, Wolff LDG. Implicações do cancelamento de cirurgias em centro cirúrgico: estudo descritivo-exploratório. Online Braz J Nurs [Internet]. 2010; [acessado 20 Julho 2021] 9(1). Disponível em: http://www.objnursing.uff.br/index.php/nursing/article/view/j.1676-4285.2010.2591/585.

3. Kurcgant P, Tronchin DMR, Melleiro, MM. A construção de indicadores de qualidade para a avaliação de recursos humanos nos serviços de enfermagem: pressupostos teóricos. Acta Paul Enferm [Internet]. 2006 jan-mar; [acessado 20 julho 2021] 19(1): 88-91. DOI: https://doi.org/10.1590/S0103-21002006000100014.

4. Sociedade Brasileira de Enfermeiros de Centro Cirúrgico, Recuperação Anestésica e Centro de Material e Esterilização (SOBECC). Diretrizes de Práticas em Enfermagem Cirúrgica e Processamento de Produtos para a Saúde. 7. ed. São Paulo: SOBECC; 2017.

5. Carvalho TA, Sobral CB, Marinho PML, Llapa-Rodriguez EOO, Campos MPA. Suspensão de cirurgias em um hospital universitário. Rev SOBECC. [Internet] 2016 out-dez; [acessado 20 julho 2021] 21(4): 186-91. DOI: https://doi.org/10.5327/Z1414-4425201600040002

6. Pinheiro SL, Vasconcelos RO, De Oliveira JLC, Matos FGOA, Tonini NS, Alves DCI. Taxa de cancelamento cirúrgico: indicador de qualidade em hospital universitário público. REME Rev Min Enferm. [Internet]2017; 21:e-1014. [acessado 20 julho 2021] DOI: DOI: http://www.dx.doi.org/10.5935/1415-2762.20170024. 
7. Moraes PGS, Pacheco NMD, Silva RGS, Silva PCV. Clinical and organizational factors related to cancellation of surgical procedures. J Nurs UFPE [Internet]. 2017; [acessado 20 julho 2021] 11(7): 2645-53. DOI: 10.5205/reuol.10939-97553-1-RV.1107201701.

8. Santos GAAC, Bocchi SCM. Cancellation of elective surgeries in a Brazilian public hospital: reasons and estimated reduction. Rev Bras Enferm. [Internet] 2017 mai-jun; [acessado 20 julho 2021] 70(3): 535-42. DOI: https://doi.org/10.1590/0034-7167-2016-0084

9. Gomes JRAA, Franco RVB, Morais DSVD, Barbosa BC. Fatores determinantes para suspensões de cirurgias eletivas em um hospital do Distrito Federal. Rev SOBECC. [Internet] 2018 out-dez; [acessado 20 julho 2021] 23(4): 184-8. DOI: https://doi.org/10.5327/Z14144425201800040003

10. Botazini NO, Toledo LD, Souza DMST. Cirurgias eletivas: cancelamentos e causas. Rev SOBECC. [Internet] 2015 out-dez; [acessado 20 julho 2021] 20(4): 210-9. DOI: http://dx.doi.org/10.5327/Z1414-4425201500040005.

11. Sampaio CEP, Gonçalves RA, Seabra Júnior HC. Determinação dos fatores da suspensão de cirurgia e suas contribuições para assistência de enfermagem. Care Online [Internet]. 2016 jul-set; [acessado 20 julho 2021] 8(3): 4813-20. DOI: http://dx.doi.org/10.9789/21755361.2016.v8i3.4813-4820.

12. Garcia ACKA, Fonseca LF. A problemática da suspensão cirúrgica: a perspectiva dos anestesiologistas. Rev Enferm UFPE [Internet]. 2013 fev; [acessado 20 julho 2021] 7(2): 48190. DOI: 10.5205/reuol.3073-24791-1-LE.0702201321

13. Risso ACMCR, Braga EM. A comunicação da suspensão de cirurgias pediátricas: sentimentos dos familiares envolvidos no processo. Rev Esc Enferm USP. [Internet]2010; [acessado 20 julho 2021] 44(2): 360-7. DOI: https://doi.org/10.1590/S008062342010000200017. 
14. Fontelles MJ, Simões MG, Farias SH, Fontelles RGS. Metodologia da pesquisa científica: diretrizes para a elaboração de um protocolo de pesquisa . Rev Para Med. [Internet] 2009 julset [acessado 20 julho 2021]; 23(3). Disponível em: http://files.bvs.br/upload/S/01015907/2009/v23n3/a1967.pdf

15. Cihoda JH, Alves JR, Fernandes LA, Souza Neto EP. Analysis for the causes of surgical cancellations in a Brazilian university hospital. Care Management J [Internet]. 2015; [acessado 20 julho 2021] 16(1): 41-7. DOI: https://doi.org/10.1891/1521-0987.16.1.41 